\title{
Combining drought and submergence tolerance in rice: marker-assisted breeding and QTL combination effects
}

\author{
Shalabh Dixit • Anshuman Singh • Nitika Sandhu • \\ Aditi Bhandari • Prashant Vikram • Arvind Kumar
}

Received: 2 March 2017 / Accepted: 9 October 2017 / Published online: 4 November 2017

(C) The Author(s) 2017. This article is an open access publication

\begin{abstract}
TDK1 is a popular rice variety from the Lao PDR. Originally developed for irrigated conditions, this variety suffers a high decline in yield under drought conditions. Studies have identified three quantitative trait loci (QTLs) for grain yield under drought conditions, $q D T Y_{3.1}, q D T Y_{6.1}$, and $q D T Y_{6.2}$, that show a high effect in the background of this variety. We report here the pyramiding of these three QTLs with SUB1 that provides 2-3 weeks of tolerance to complete submergence, with the aim to develop drought- and submergence-tolerant near-isogenic lines (NILs) of TDK1. We used a tandem approach that combined marker-assisted backcross breeding with phenotypic selection to develop NILs with high yield under drought stress and non-stress conditions and preferred grain quality. The effect of different QTL combinations on yield and yield-related traits
\end{abstract}

Electronic supplementary material The online version of this article (https://doi.org/10.1007/s11032-017-0737-2) contains supplementary material, which is available to authorized users.

S. Dixit $\cdot$ A. Singh $\cdot$ N. Sandhu $\cdot$ A. Bhandari $\cdot$ P. Vikram $\cdot$

A. Kumar $(\square)$

International Rice Research Institute (IRRI), DAPO Box 7777,

Metro Manila, Philippines

e-mail: a.kumar@irri.org

A. Singh

Rani Lakshmi Bai Central Agriculture University, Jhansi, India

P. Vikram

International Maize and Wheat Improvement Center (CIMMYT), Km. 45, Carretera México-Veracruz, El Batán, 56237 Texcoco, $\mathrm{CP}$, Mexico under drought stress and non-stress conditions is also reported. Our results show $q D T Y_{3.1}$ to be the largest and most consistent QTL affecting yield under drought conditions, followed by $q D T Y_{6.1}$ and $q D T Y_{6.2}$, respectively. QTL class analysis also showed that lines with a combination of $q D T Y_{3.1}$ and $q D T Y_{6.1}$ consistently showed a higher tolerance to drought than those in which one of these QTLs was missing. In countries such as Lao PDR, where large areas under rice cultivation suffer vegetative-stage submergence and reproductive-stage drought, these lines could ensure yield stability. These lines can also serve as valuable genetic material to be used for further breeding of high-yielding, drought- and submergence-tolerant varieties in local breeding programs.

Keywords Rice · Drought - Yield · Submergence - QTL . MABB

\section{Introduction}

Drought and flooding are considered to be two of the most important abiotic stresses that affect rice production globally. A total of approximately 40 million hectares of rice area are affected by different forms of the two stresses occurring at different crop stages. Under natural conditions, both of these stresses are expected to occur at different levels in the topographic sequence. However, in rainfed rice ecosystems, these two stresses are often observed to occur in the same area within a 
growing season. This combination of stresses is especially relevant in South and Southeast Asia where rainfed rice ecosystems are major natural disaster hotspots prone to drought and flood risks (Dilley et al. 2005). For example, in eastern India, the onset of the monsoon may bring heavy rains in July-August that cause flash floods at the vegetative stage of the rice crop. However, early withdrawal of the monsoon or prolonged dry phases at the reproductive stage may cause considerable yield loss due to drought. Similarly, in the Mekong River basin in Southeast Asia, rainfed areas in countries such as Lao PDR, Thailand, Vietnam, and Cambodia are prone to submergence and drought within or across seasons and locations. Among these countries, Lao PDR is likely the most affected by drought and flood. The rainfed lowland ecosystem dominates rice cultivation in the country and is highly prone to natural disasters. In 2004, rainfed lowlands accounted for about $75 \%$ of the total area and $78 \%$ of the production (www.irri.org). Although floods are a common phenomenon in this region due to the presence of the Mekong River, an increase in drought incidences has also been observed in the past two decades (Komany 2004). TDK1 is a popular rice variety that is cultivated in a large part of the rainfed lowland ecosystems in Lao PDR. A submergence-tolerant version of this variety (TDK1-Sub1) has been developed which provides considerable tolerance to flash floods; however, both TDK1 and TDK1-Sub1 are highly susceptible to drought. SUB1 is a major quantitative trait locus (QTL) derived from landrace FR13A that provides tolerance of 23 weeks of complete submergence (Septiningsih et al. 2014). This QTL was found to account for $69 \%$ of the phenotypic variance in the original identification study (Xu and Mackill 1996) and has been used extensively in marker-assisted backcross breeding (MABB) programs to improve mega-varieties with tolerance of submergence (Neeraja et al. 2007; Septiningsih et al. 2014; Toledo et al. 2015).

Similar to the advances made in submergence tolerance, considerable progress has also been made in understanding the genetics of grain yield under drought conditions in the past decade at the International Rice Research Institute (IRRI). Selection for grain yield under drought conditions has been used as an effective breeding strategy (Kumar et al. 2009; 2013; 2014; Dixit et al. 2014b). Several large-effect QTLs have also been reported for the trait in studies conducted on a wide range of mapping populations (Bernier et al. 2007;
Venuprasad et al. 2009; Vikram et al. 2011; Ghimire et al. 2012; Swamy et al. 2013; Yadav et al. 2013; Mishra et al. 2013; Palanog et al. 2014; Sandhu et al. 2010; Dixit et al. 2014a, c, b). In addition to explaining a large proportion of the phenotypic variances for grain yield under drought, many of these QTLs have shown consistency of effect across genetic backgrounds. Further, studies targeting more detailed analysis of these QTLs to understand epistatic effects and QTL physiology or for fine mapping and candidate gene identification of some of these QTLs have also been conducted (Henry et al. 2014; Dixit et al. 2012a, b; 2015a). Three such QTLs, namely, $q D T Y_{3.1}, q D T Y_{6.1}$, and $q D T Y_{6.2}$, were identified in the background of TDK1 with large effects on grain yield under drought conditions (Dixit et al. 2014c). $q D T Y_{3.1}$ was identified as the most consistent QTL, with an effect across two levels of stress under lowland conditions and in upland mild stress conditions, and $q D T Y_{6.1}$ and $q D T Y_{6.2}$ showed effects under lowland severe stress and upland mild stress conditions (Dixit et al. 2014c).

The initial aim of this study was to pyramid $q D T Y_{3.1}$, $q D T Y_{6.1}$, and $q D T Y_{6.2}$ with SUB1 to develop highyielding drought- and submergence-tolerant near-isogenic lines (NILs) of TDK1 with preferred grain quality. We report a tandem selection approach combining MABB and phenotypic selection that not only allowed us to combine the four QTLs (3 drought QTLs and Sub1) together in TDK1 but also allowed us to identify lines with high yield and preferred grain quality. We also report the effect of different combinations of DTY QTLs on yield and yield-related traits under drought stress and non-stress conditions. The NILs identified from this study will be advanced further and tested in Lao PDR and other countries of Southeast Asia for possible release and distribution in rainfed areas as a replacement for TDK1.

\section{Material and methods}

This study was conducted at the Ziegler Experiment Station of the IRRI, Los Baños, Laguna, Philippines. Marker-assisted backcrossing, advancement, and fixing of lines were carried out from 2012 to 2016. The drought stress, non-stress, and submergence experiments for NILs were conducted in the dry season (DS) and wet season (WS) of 2015. 
Plant material

$q D T Y_{3.1}, q D T Y_{6.1}$, and $q D T Y_{6.2}$ were identified in a $\mathrm{BC}_{1} \mathrm{~F}_{3: 5}$ population derived from the cross of IR55419-04 and TDK1 (Dixit et al. 2014c). TDK1 is a popular indica variety that covers a large area under the rainfed ecosystem in Lao PDR. This variety was developed from the cross SPT 7149-429-3/IR13423$10-2-3$. It is a long-duration variety with high tillering and yield potential, but it shows high susceptibility to drought and submergence. In comparison, IR55419-04 is a drought-tolerant line developed from the cross IR12979-24-1/UPLRi 5. It is a medium-duration indica line with high yield potential and tolerance of drought. Lines from the mapping population showing the presence of the full segment of the three QTLs were used as the donors for the backcross program. TDK1-Sub1, the submergence-tolerant version of TDK1, was used in the backcross program as a recurrent parent to pyramid the three drought QTLs with SUB1.

Genotypic data and development of chromosome maps

Rice simple-sequence repeat (SSR) markers were used for foreground selection throughout the crossing program while background recovery check at the final stage of the program was done using singlenucleotide polymorphism (SNP) markers. The SSR markers closely linked to the QTLs are given in Electronic Supplementary Material (ESM) Table 1, and the SSR genotyping protocol is presented in ESM file 1. SNP genotyping was conducted using the Infinium $6 \mathrm{~K}$ SNP genotyping platform (Illumina Inc., San Diego, CA) for the selected NILs. Gamma-glutamyltransferase 2 (GGT2) (Van Berloo 2008) was used for the construction of chromosome maps of NILs. SNP data also provided information on polymorphic SNPs within each QTL region that can be used for marker-assisted selection (MAS) in future studies. A list of these SNPs is provided in ESM file 2.

\section{QTL introgression and selection}

The crossing scheme used to pyramid $q D T Y_{3.1}, q D T Y_{6.1}$, $q D T Y_{6.2}$, and SUB1 into TDK1 is given in ESM Fig. 1. Seventeen $\mathrm{BC}_{1} \mathrm{~F}_{5}$ lines with full segments of the three QTLs were selected from the mapping population and crossed to TDK1-Sub1 to develop a large $\mathrm{BC}_{2} \mathrm{~F}_{1}$ population. A total of $658 \mathrm{BC}_{2} \mathrm{~F}_{1}$ plants were genotyped with foreground markers, and plants segregating for the three QTLs were selected. These plants were used to develop a large $\mathrm{BC}_{2} \mathrm{~F}_{2}$ population (approx. 10,000 plants). From this stage onward, a tandem marker-assisted and phenotypic selection approach was used to advance the lines. First, the $\mathrm{BC}_{2} \mathrm{~F}_{2}$ population underwent phenotypic selection for TDK1 plant type, resulting in the selection of 5700 plants. The selected plants were then genotyped with the foreground markers, and plants with different combinations of QTLs and SUB1 were identified. A $\mathrm{BC}_{2} \mathrm{~F}_{3}$ population of 834 lines was developed from the selected plants for further purification and testing. This population was screened under drought stress and nonstress conditions, and a total of 228 single plants were selected and advanced to the $\mathrm{BC}_{2} \mathrm{~F}_{4}$ generation. These selected $\mathrm{BC}_{2} \mathrm{~F}_{4}$ lines were tested again under severe drought stress conditions, and further selections were conducted. A total of 44 lines were advanced to the $\mathrm{BC}_{2} \mathrm{~F}_{5}$ generation through single-plant selections for seed multiplication and estimation of yield potential under non-stress conditions. In addition, plant selections were conducted from all 44 lines to generate $\mathrm{BC}_{2} \mathrm{~F}_{6}$ pure lines for screening under drought, submergence, and non-stress conditions. A total of 105 panicles were also selected from this population based on their similarity to TDK1 or TDK1-Sub1; these were used to develop a parallel set of $105 \mathrm{BC}_{2} \mathrm{~F}_{6}$ with grain type similar to that of the recipients. A combined set of $42 \mathrm{BC}_{2} \mathrm{~F}_{7}$ lines selected from this set and ten $\mathrm{BC}_{2} \mathrm{~F}_{7}$ lines selected from the first set was then screened under drought stress and non-stress conditions. Twenty-nine $\mathrm{BC}_{2} \mathrm{~F}_{8}$ lines were selected, and these lines underwent a final testing in replicated trials for yield under drought and non-stress conditions and for submergence tolerance. A set of ten lines was identified from this trial to test further in Lao PDR. Since the major target in this study was to develop high-yielding, drought-tolerant lines with different combinations of DTY QTLs and SUB1 and with grain quality similar to that of TDK1, the lines were selected based on the foreground markers throughout the selection process. However, to estimate the recovery of the recipient genome in selected lines obtained through MAS and phenotyping results, background genotyping was conducted on selected $\mathrm{BC}_{2} \mathrm{~F}_{6}$ lines using SNP markers (4606 SNPs). All lines showed $>90 \%$ recovery of the TDK1 genome (ESM Fig. 2). However, higher background similarity could have been achieved if the selection focus would not be overall phenotypic performance after QTL introgression. 
Management of drought and non-stress experiments

Near-isogenic lines selected at various stages of the backcross program were screened under reproductivestage drought stress in a rainout shelter facility, while non-stress experiments were conducted in open fields at IRRI. All trials were conducted in the lowland transplanted ecosystem. In the DS of 2015 (DS-2015), the lines were planted in an alpha lattice design with two replications and a plot size of $0.7 \mathrm{~m}^{2}$ for the drought stress experiment, and in a single replication in an augmented randomized complete block design with a plot size of $1 \mathrm{~m}^{2}$ for the non-stress experiment. In the WS of 2015 (WS-2015), both the stress and non-stress experiments were conducted in an alpha lattice design in two replications. The plot size was 1.4 and $2.0 \mathrm{~m}^{2}$ under stress and non-stress conditions, respectively. Smaller plot sizes were maintained due to the limited amount of seeds harvested from plant selections in the previous season. In DS-2016, both stress and non-stress trials were planted in an alpha lattice design with two replications and plot sizes of 2.9 and $4 \mathrm{~m}^{2}$, respectively. Field and crop management was conducted as outlined by Dixit et al. (2014c).

In all experiments, days to flowering (DTF), plant height at maturity $(\mathrm{PH})$, and grain yield (GY) were recorded (see Dixit et al. 2014c for the detailed procedure). Grain length and width for ten whole and de-hulled grains were measured using a Vernier caliper and the length-width ratio calculated. Kernel shape was determined using the length-width ratio as described in the standard evaluation system for rice (IRRI 2014). There were 100 grains for each NIL, and TDK1 and TDK1-Sub1 grains were sampled randomly from the seed lot and weighed to determine the grain weight.

\section{Management of submergence experiments}

Submergence screening was conducted at seedling stage in DS-2015 and WS-2015. Selected NILs were planted in nursery beds along with TDK1 and TDK1-Sub1 and the susceptible check IR42. The lines were allowed to grow without submergence up to 14 days and then were submerged. The fields were drained after 13 days based on the survival of the susceptible check IR42. Final recovery was recorded at 7 days after draining. The lines were scored based on the standard evaluation system for rice for submergence tolerance on a scale of 1-9 in increasing order of susceptibility. Submergence screening in DS-2016 was conducted in a concrete tank facility. The lines were seeded in trays and submerged at 14 days after sowing (DAS) along with the susceptible check. The lines were de-submerged after 16 days based on the survival of the susceptible check IR42. The actual number of seedlings per line was recorded before desubmergence and 2, 7, 14, and 21 days after de-submergence, and the percentage survival was calculated.

\section{Statistical analysis}

The data of all experiments were analyzed using CROPSTAT version 7.2.3 (http://archive.irri. org/science/software/cropstat.asp). Mixed model analysis of the data was carried out using the model

$y_{i j k}=\mu+g_{i}+r_{j}+b_{l j}+e_{i j k}$

where $\mu$ is the overall mean, $g_{i}$ is the effect of the $\mathrm{i}$-th genotype, $r_{k_{j}}$ is the effect of the $\mathrm{j}$-th replicate, $b_{l k}$ is the effect of the 1-th block within the $\mathrm{j}$-th replicate, and $e_{i j k}$ is the error. Genotype effects were considered to be fixed and the replicates and block effects to be random.

\section{Results}

Phenotypic variance

The phenotypic variance observed among the parents and progenies in field experiments and the submergence experiment is summarized in Table 1. Significant variance for all traits was observed under drought stress and non-stress conditions except for PH in DS-2015 (Table 1). IR55419-04 showed higher tolerance and significantly high GY under drought conditions in all experiments compared with TDK1 and TDK1-Sub1. TDK 1 and TDK1-Sub1 outyielded IR55419-04 in DS-2015 and DS-2016 in the non-stress experiment. In WS-2015, an opposite pattern of yield under nonstress conditions was observed, in which IR55419-04 showed higher yield than the other two parents (Table 1); the difference was non-significant in DS2015 and WS-2015, while a significant difference was observed in DS-2016 for the trait. The mean yield of the progenies remained between the high- and low-yielding parents in all experiments. IR55419-04 was the earlyflowering parent among the three, while TDK1 and TDK1-Sub1 showed a similar flowering time under 


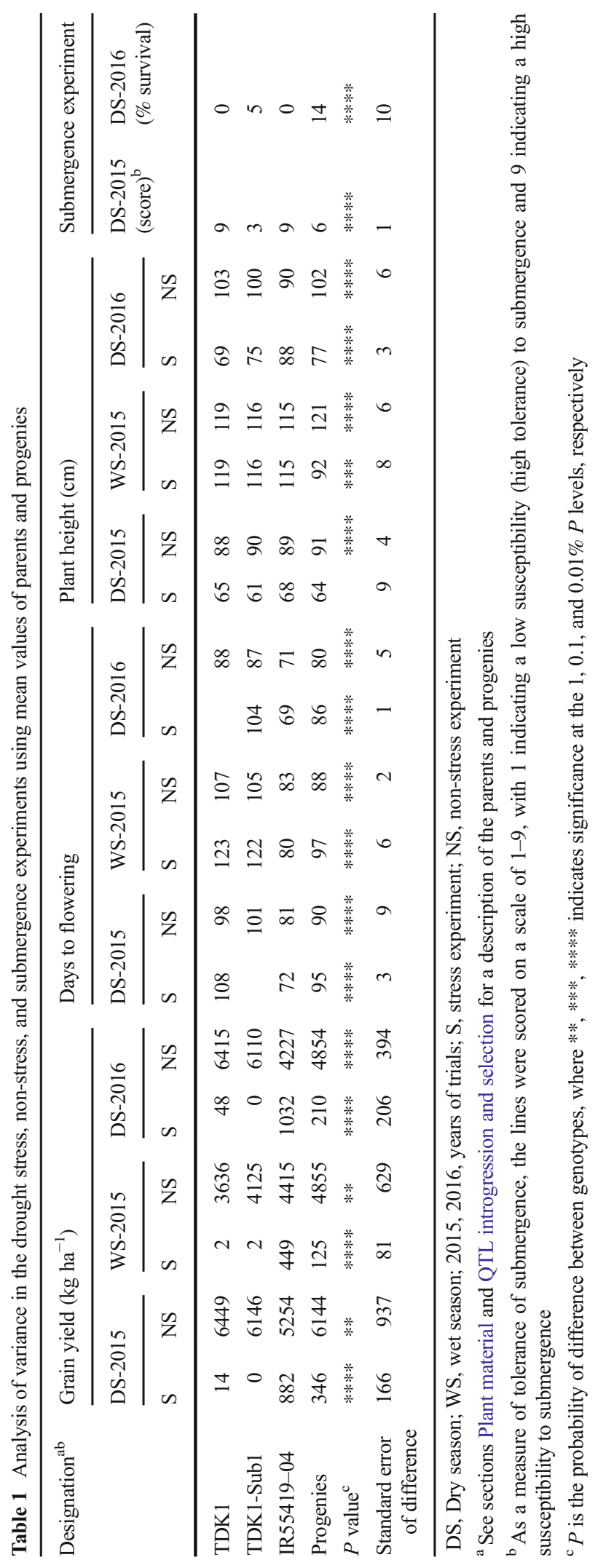


both stress and non-stress conditions. The progeny means lay between IR55419-04 and the two lateflowering parents. All three parents showed similar PH in all experiments, except in DS-2016 when IR5541904 had lower PH than the other two parents under the non-stress conditions. However, a higher reduction in PH was observed for TDK1 and TDK1-Sub1 under the stress conditions. The mean $\mathrm{PH}$ of the progenies was also similar to that of the recipient parents under drought stress and non-stress conditions. TDK1 and IR5541904 showed high susceptibility to submergence compared with the tolerant donor TDK1-Sub1; the progeny mean fell between those of the tolerant parent and the two susceptible parents (Table 1).

\section{Trait correlations and QTL class analysis}

The phenotypic correlations between GY, PH, and DTF under drought stress and non-stress conditions in DS2015 are presented in Table 2. GY under stress conditions showed significant negative correlations with DTF, thereby revealing the advantage of earlyflowering lines under stress conditions. However, a positive correlation between GY and DTF was observed under non-stress conditions. Apart from this, a significant negative correlation between stress and non-stress in GY was also observed. No significant correlations were observed between GY and PH under drought conditions; however, under non-stress conditions, the two traits were positively correlated.

In order to understand the correlations of the three traits at the genetic level and to understand the effect of the three $D T Y$ on these traits, a QTL class analysis was conducted with $q D T Y_{3.1}, q D T Y_{6.1}$, and $q D T Y_{6.2}$ (Fig. 1). The class analysis revealed an effect of $q D T Y_{3.1}$ on DTF of the lines (Fig. 1a). The two classes of lines with this QTL [+++ (possessing all 3 QTLs) and +-+ (possessing $q D T Y_{3.1}$ and $\left.q D T Y_{6.2}\right]$ showed early flowering compared with the two without them $[-++$ (possessing $q D T Y_{6.1}$ and $q D T Y_{6.2}$ ) and --+ (with $q D T Y_{6.2}$ only)]. $q D T Y_{6.1}$ was also observed to play a role along with $q D T Y_{3.1}$ in causing earliness in the lines. It was observed that lines with both these QTLs (+++) flowered much earlier than those with one of the two QTLs (+-+ and $-++)$ and that the lines without the two QTLs [--+ (possessing $q D T Y_{6.2}$ only) flowered even later. TDK1 [---, without any of the 3 QTLs) flowered the latest of all classes. For GY (Fig. 1b), a similar relation was observed under drought conditions, for which lines with both $q D T Y_{3.1}$ and $q D T Y_{6.1}(+++)$ showed higher GY than those with one of the two QTLs (-++ and +-+). $q D T Y_{3.1}$, however, had a larger effect on GY than $q D T Y_{6.1}$, as a smaller difference from the trial mean was observed for class +-+ than for class -++ . These two classes were followed by the last class with $q D T Y_{6.2}$ only (-+), whereas all classes yielded higher GY under drought conditions than TDK1 (- - ). $q D T Y_{3.1}$ caused a reduction in GY under non-stress conditions as the QTL classes +++ and +-+ showed lower mean yields than the trial mean. In the case of $\mathrm{PH}, q D T Y_{6.1}$ seemed to be the only QTL showing an effect under non-stress conditions as classes +++ and -++ consistently showed positive differences from the trial mean (Fig. 1c). No clear pattern of effect of the other two QTLs was observed for PH.

Selected lines, their performance, and grain type

These results showed the advantage of $q D T Y_{3.1}$ under stress conditions and its disadvantage under non-stress conditions. Apart from this, the program also targeted the identification of lines tolerant of submergence and drought and maintenance of TDK1/TDK1-Sub1 grain quality. Because of this, phenotypic selection at the end of the MAS program became very important to identify high-yielding drought-tolerant lines. The identified lines screened under drought stress and non-stress conditions
Table 2 Phenotypic correlations between traits under drought stress and non-stress conditions in DS-2015

*, **Significant at the 5 and $1 \%$ levels of significance, respectively

DTF, Days to flowering; PH, plant height at maturity; GY, grain yield

\begin{tabular}{lcccccc}
\hline Traits & DTF-NS & PHN-S & GY-NS & DTF-S & PH-S & GY-S \\
\hline DTF-NS & 1 & & & & \\
PH-NS & -0.12 & 1 & & & \\
GY-NS & $0.31^{*}$ & $0.50^{* *}$ & 1 & & \\
DTF-S & $0.84^{* *}$ & -0.18 & $0.31^{*}$ & 1 & 1 & \\
PH-S & -0.14 & $0.36^{*}$ & 0.07 & -0.20 & 0.13 & 1 \\
GY-S & $-0.74^{* *}$ & 0.08 & $-0.31^{*}$ & $-0.88^{* *}$ & \\
\hline
\end{tabular}




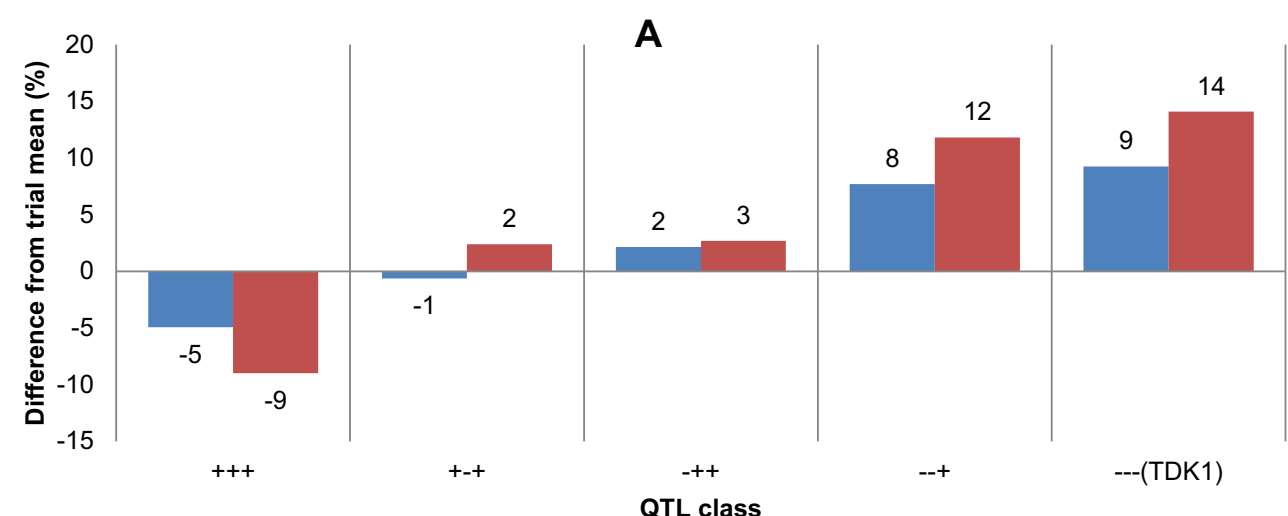

non-stress $\quad$ Stress

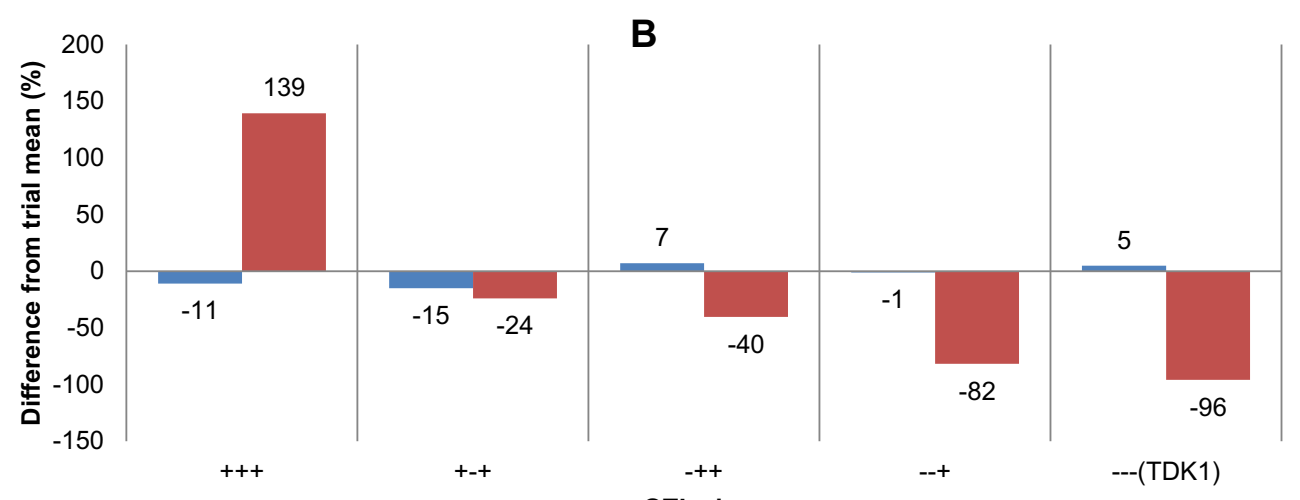

QTL class

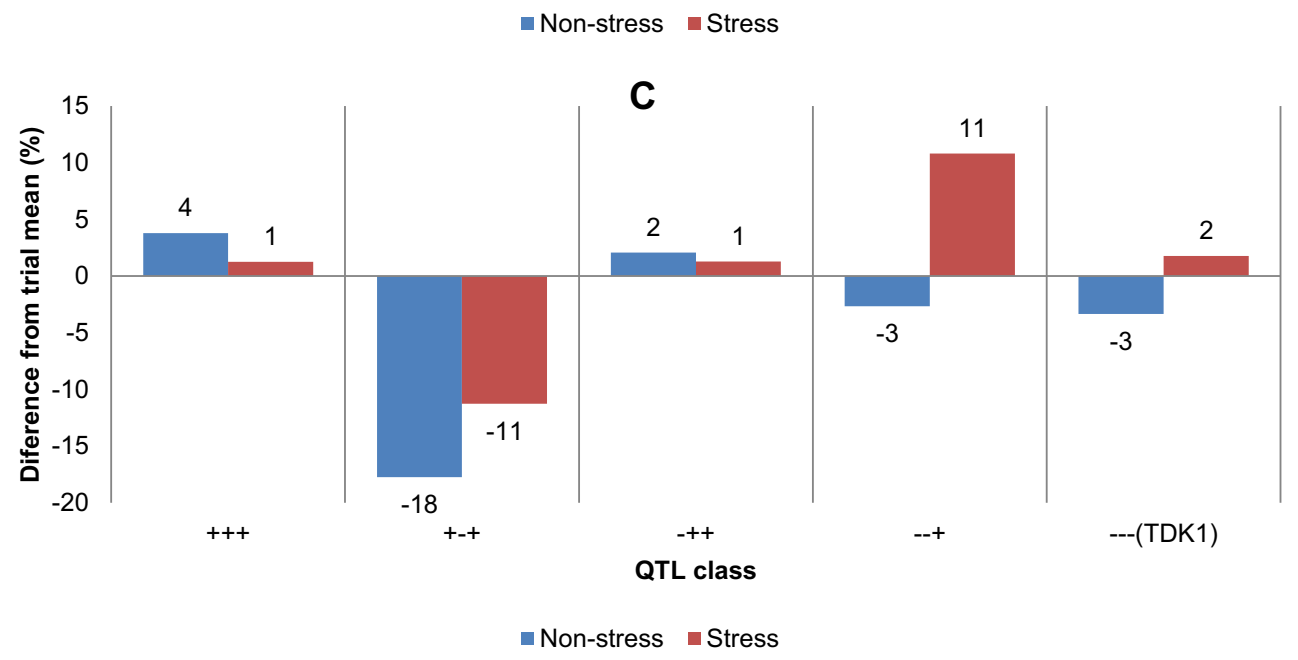

Fig. 1 Effect of combinations of $q D T Y_{3.1}, q D T Y_{6.1}$, and $q D T Y_{6.2}$, 3 quantitative trait loci (QTLs) identified in the background of rice variety TDK1 with large effects on grain yield under drought conditions, on a days to flowering, b grain yield (GY) and c plant height under drought stress and non-stress conditions. +++ class refers to lines with all 3 QTLs, +-+ class refers to lines with $q D T Y_{3.1}$ and $q D T Y_{6.2},-++$ class refers to lines with $q D T Y_{6.1}$ and $q D T Y_{6.2},--+$ class refers to lines with $q D T Y_{6.2}$ only; TDK1 (-is taken as the baseline for lines without any QTLs. Lines with three QTLs showed the highest yield advantage under drought conditions and earlier flowering and maintained plant height. $q D T Y_{3.1}$ led to a yield penalty under non-stress conditions 
showed varying performance for the two stresses and GY under non-stress conditions. Of the 44 lines screened, 15 lines showed high GY under drought conditions; however, only nine of these 15 lines showed high GY under non-stress conditions and only seven of these same 15 lines showed tolerance of both drought and submergence (Fig. 2). Similarly, of the 19 lines with high GY potential under non-stress conditions, ten showed tolerance of submergence. We identified three lines in which all three traits were successfully combined. These lines showed increased tolerance of submergence along with high GY under drought conditions compared with TDK1 and more than/similar GY as TDK1 under non-stress conditions. The performance of seven selected lines is shown in Table 3. Four of these lines showed high GY under drought stress and non-stress conditions compared with TDK1; however, these lines showed similar susceptibility to submergence as TDK1. The other three lines showed higher tolerance of both stresses than TDK1 and high GY. In DS-2015, the mean GY of six of the seven lines ranged between the mean of the tolerant parent IR55419-04 and that of the susceptible parent TDK1. However, IR102776-31-

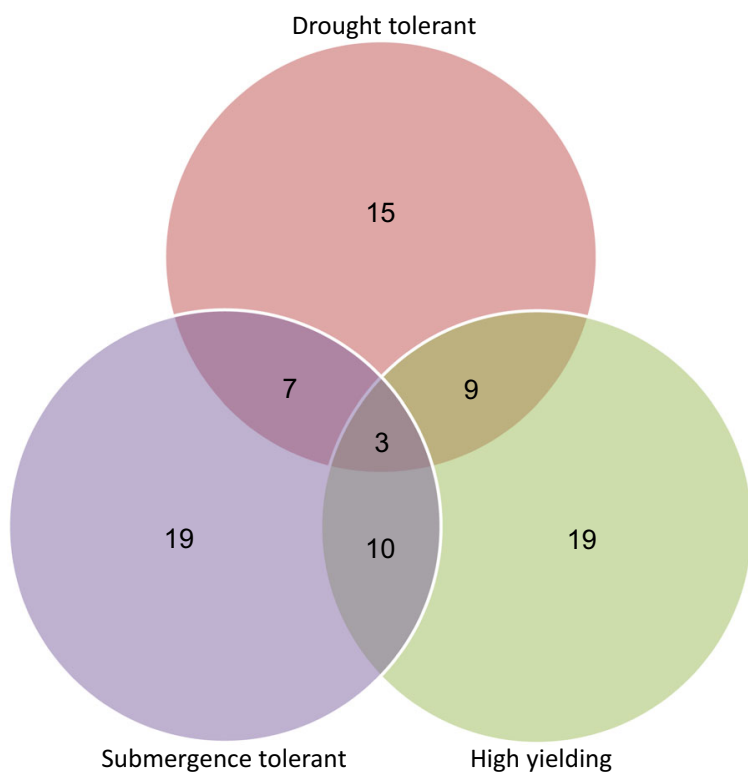

Fig. 2 Vann diagram showing the proportion of lines with tolerance of drought and submergence and with high yield from the set of lines screened in the dry season of 2015. Numbers in the circles refer to lines with one of the three traits while those in the intersecting areas show the lines with two or all three traits. In total, 29 lines showed the presence of a combination of two or three traits
66-2-2-2 showed higher GY than the tolerant parent IR55419-04. In WS-2015, a much more severe stress was imposed than in DS-2015. A similar pattern of GY was observed for the lines, with mean yields ranging between those of the tolerant and susceptible parents. Although the differences in GY became less apparent in this season due to the high severity of stress, three of the lines still showed significantly higher GY than TDK1. The two susceptible parents showed almost no GY in both seasons (Table 3). A second set of lines was also identified from DS-2015 screening (ESM Table 2). These lines were those that showed relatively moderate GY under drought stress conditions compared with the first set of lines, but they had a grain type much closer to that of TDK1-Sub1.

Grain measurements were conducted for all the identified lines. In general, the grain shape of all lines was close to each other. and a majority of them, including the recurrent parent TDK1 and TDK1Sub1, were classified as slender grain types (ESM Table 3a). However, IR 102774-11-128-1-4-3 showed medium grain type that was similar to that of the donor parent IR55419-04. All lines with SUB I showed a similar light-colored hull as TDK1-Sub1, whereas those without SUB1 showed a similar hull color as TDK1. An exception to this was line IR102777-5-64-4-1-5, which had a white hull color despite lacking $S U B 1$; this can be attributed to an effect from the donor line IR55419-04, which also has light hull color. All seven lines selected based on GY, tolerance of drought and submergence, and QTL presence were similar to the recipient in terms of grain measurements. They all showed intermediate chalkiness between TDK1 and IR55419-04 (ESM Fig. 3a). However, the second set of lines was much closer to the recipient parent in terms of grain shape and waxiness (ESM Table 3b; ESM Fig. 3b). During the MABB program, we also created pure lines based on panicle selection conducted for grain type. These were tested in DS-2016 in large plots along with the previously identified lines. The performance of these lines is presented in ESM Table 4. We identified a total of seven lines from this set that showed tolerance of drought and submergence. Consistent with previous seasons, IR102776-31-66-2-2-2 showed high yield under drought conditions and tolerance of submergence (ESM Table 4). All lines showed similar grain structure as the recipient, and IR102777-18128-2-1-4 also had high waxiness. 
Table 3 Selected near-isogenic lines and their grain yield, days to 50\% flowering, and plant height under drought stress and non-stress conditions in DS-2015 and WS-2015 and under submergence conditions in DS-2015

\begin{tabular}{|c|c|c|c|c|c|c|c|c|c|c|c|c|c|c|}
\hline \multirow[t]{3}{*}{ Line } & \multicolumn{6}{|c|}{ DS-2015 } & \multicolumn{5}{|c|}{ WS-2015 } & \multirow{3}{*}{$\begin{array}{l}\text { Submergence } \\
\text { S }\end{array}$} & \multirow{2}{*}{\multicolumn{2}{|c|}{ QTL }} \\
\hline & \multicolumn{2}{|c|}{$\mathrm{GY}\left(\mathrm{kg} \mathrm{ha}^{-1}\right)$} & \multicolumn{2}{|c|}{ DTF } & \multicolumn{2}{|c|}{$\mathrm{PH}(\mathrm{cm})$} & \multicolumn{2}{|c|}{ GY $\left(\mathrm{kg} \mathrm{ha}^{-1}\right)$} & \multirow{2}{*}{$\begin{array}{l}\text { DTF } \\
\text { NS }\end{array}$} & \multicolumn{2}{|c|}{$\mathrm{PH}(\mathrm{cm})$} & & & \\
\hline & NS & $\mathrm{S}$ & NS & $\mathrm{S}$ & NS & $\mathrm{S}$ & NS & $\mathrm{S}$ & & S & NS & & B & $\mathrm{L}$ \\
\hline IR102774-11-128-1-4-3 & 6773 & 466 & 86 & 88 & 98 & 73 & 5244 & 182 & 87 & 84 & 129 & 101 & 9 & $\mathrm{H}++$ \\
\hline IR102776-37-52-1-1-3 & 6760 & 534 & 86 & 89 & 105 & 81 & 5050 & 300 & 86 & 82 & 132 & 106 & 9 & -++ \\
\hline IR102775-24-97-1-1-1 & 6448 & 904 & 90 & 87 & 84 & 70 & 4305 & 95 & 87 & 96 & 107 & 78 & 9 & +++ \\
\hline IR102777-5-64-4-1-5 & 6656 & 595 & 87 & 88 & 84 & 60 & 4144 & 238 & 86 & 88 & 108 & 91 & 9 & $+\mathrm{H}-$ \\
\hline IR102776-31-66-2-2-2 & 6615 & 1173 & 87 & 87 & 94 & 65 & 5533 & 58 & 88 & 93 & 117 & 80 & 6 & +++ \\
\hline IR102774-15-32-3-1-2 & 7255 & 718 & 87 & 91 & 110 & 66 & 5511 & 40 & 84 & 91 & 132 & 104 & 5 & +++ \\
\hline IR102777-5-83-1-2-2 & 7112 & 519 & 87 & 89 & 101 & 82 & 4897 & 37 & 90 & 104 & 132 & 92 & 4 & -++ \\
\hline IR55419-04 & 5254 & 882 & 81 & 72 & 89 & 68 & 4415 & 449 & 83 & 80 & 115 & 100 & 9 & \\
\hline TDK1 & 6449 & 14 & 98 & 108 & 88 & 65 & 3636 & 2 & 107 & 123 & 119 & 80 & 9 & \\
\hline TDK1-Sub1 & 6146 & 4 & 101 & & 90 & 61 & 4125 & 2 & 105 & 122 & 116 & 83 & 3 & \\
\hline
\end{tabular}

\section{Discussion}

Our study reports the development of drought- and submergence-tolerant versions of a popular rice variety, TDK1, from Lao PDR and the effect of different combinations of DTY QTLs on the performance of lines under drought and non-stress conditions. NILs of TDK1 with different combinations of $q D T Y_{3.1}, q D T Y_{6.1}$, and $q D T Y_{6.2}$ and $S U B 1$ were developed and tested under drought stress, non-stress, and submergence conditions to identify the best NILs and QTL combinations. The study also aimed at determining the effect of combining the DTY QTLs with $S U B 1$ on the performance of lines. $q D T Y_{3.1}, q D T Y_{6.1}$, and $q D T Y_{6.2}$ were identified in a $\mathrm{BC}_{1}$-derived population developed from the cross of IR55419-04 and TDK1 (Dixit et al. 2014c). We crossed lines from this mapping population to TDK1-Sub1 to develop a $\mathrm{BC}_{2}$ population segregating for all three $D T Y$ QTLs and SUB1. Maintenance of grain quality, plant type, and yield potential is of the utmost importance while targeting the development of a product for commercial release and cultivation. In this case, it was required to develop lines with the same or higher grain quality and GY than the recipient variety TDK1 or TDK1-Sub1. Apart from this, the QTL with the largest effect on GY under drought $\left(q D T Y_{3.1}\right)$ targeted in this study is known to reduce GY under non-stress conditions (Venuprasad et al. 2009). In order to eliminate any undesirable effects on other traits and develop high- yielding lines with TDK1 or TDK1-Sub1 grain type, we applied a tandem selection approach using both genotypic data and phenotypic selection to advance the lines. A similar approach has been described by Han et al. (1997) in barley. However, in our case, we targeted phenotypic selection for different traits at different stages of the MAS to maximize the genetic gain (ESM Fig. 1). The screening under drought stress, nonstress, and submergence conditions revealed that there was high variation among the parents and progenies (Table 1), which allowed the selection of lines with all/ most of the desired characters. This variation may have resulted from positive interaction between QTLs or interaction of one or more QTLs with the genetic background (Dixit et al. 2014c). The analysis of the effect of DTY QTLs on GY, DTF, and PH showed a high positive effect of $q D T Y_{3.1}$ on GY under drought conditions, whereas a negative effect of this QTL was observed under non-stress conditions (Fig.1). A similar response of this QTL has been reported previously in studies in which this QTL was identified (Venuprasad et al. 2009; Dixit et al. 2014c). Apart from this, similar to Dixit et al. ( 2014c), a complementary effect of $q D T Y_{3.1}$ and $q D T Y_{6.1}$ was observed in this study. and $q D T Y_{6.2}$ showed a relatively smaller effect. The similarity and consistency of the effect patterns of these two QTLs in the original mapping population and NIL trials not only show their suitability for MABB but also show that the presence of the $S U B 1$ gene does not alter the effect 
pattern of DTY QTLs in this specific case. Despite the overall negative effect of $q D T Y_{3.1}$ on GY under nonstress conditions, we were able to identify NILs with yield potential similar to or more than TDK1 and TDK1Sub1. A total of seven high-yielding NILs were ultimately identified after the first two seasons of screening (Table 3). Four of these showed higher tolerance of drought while the other three showed tolerance of drought and submergence compared with the parent TDK1 (Table 3). The association of RM468, one of the foreground markers of this QTL, has been reported previously to have a negative effect on GY under nonstress conditions (Dixit et al. 2014c). In our study, of the 44 NILs tested under drought stress and non-stress conditions at the end of the MAS program, two highyielding NILs with a full segment of $q D T Y_{3.1}$ were identified. This result suggests a recombination event leading to the elimination of the factors causing the negative effect on GY due to continuous phenotypic selection coupled with MAS. In terms of grain quality, two sets of NILs were identified: the first set included those with intermediate waxiness (ESM Fig. 3a), while the second included those with high waxiness similar to that of TDK1 and TDK1-Sub1 (ESM Fig. 3b). All except one of the identified NILs showed similar grain shape as TDK1 and TDK1-Sub1 (ESM Table 3; ESM Fig. 3). TDK1 has a dark-colored hull and TDK1-Sub1 has a light-colored hull. This variation in hull color has also been observed in other cases, such as for the Indian rice variety Swarna, for which the SUB1 introgression has led to a light-colored hull. In our study, all lines with SUB1 showed similar light-colored hull as TDK1-Sub1, whereas the majority of those without $S U B 1$ showed a similar hull color as TDK1. These features of the two sets of NILs are very important to identify their potential use and target environment for release as varieties. While the first set can be a suitable breeding material for high tolerance of drought and submergence and further increasing waxiness, the second set of NILs can be suitable for release in drought- and submergence-prone areas where waxiness is preferred. Among all lines evaluated, IR102776-31-66-2-2-2 looks to be outperforming other lines, as was also confirmed in WS-2016 experiments in drought and non-stress conditions.

MAS has been used extensively in rice breeding to pyramid genes/QTLs of interest in popular rice cultivars. Several studies have targeted genes conferring tolerance of major biotic stresses to successfully develop tolerant lines through MAS (Huang et al. 1997;
Hittalmani et al. 2000; Singh et al. 2001). Similarly, QTLs/genes underlying GY-related traits and grain quality in rice have also been used for MAS (Joseph et al. 2004; Zhang et al. 2006; Yi et al. 2009). For abiotic stress tolerance in rice, some famous examples of successful MAS are of the submergence tolerance genes SUB1 (Neeraja et al. 2007) and SNORKEL 1 (Hattori et al. 2009), Saltol for salinity tolerance (Linh et al. 2012), DTY QTLs (Swamy et al. 2013; Dixit et al. 2015a, b; Shamsudin et al. 2016a, b), and Dro 1 (Uga et al. 2013) for drought tolerance. However, studies on combining tolerance of two major abiotic stresses through MAS are very rare. Our study has successfully demonstrated that, through a systematic MAS program combined with phenotypic selection, not only tolerance of multiple stresses can be achieved but also other important characters such as yield potential, plant type, and grain quality can be retained. In countries such as Lao PDR, where large areas under rice cultivation suffer vegetative-stage submergence and reproductive-stage drought, these lines could prove to be an efficient tool to ensure yield stability. These lines can also serve as valuable genetic material to be used for further breeding of high-yielding drought- and submergence-tolerant varieties in local breeding programs.

Acknowledgements The authors thank the Bill \& Melinda Gates Foundation for providing financial support for this study. The authors also acknowledge the technical support received from Jhon Ontoy, Margaret Catolos, Joahna Ruth Ramirez, Ma. Teresa Sta Cruz, Paul C. Maturan, Teodoro Cuevas, Marcial Panting, Jocelyn Guevarra, Carmela Malabanan, Ariel Formarejo, Ruth Erica Carpio, and Jennylyn Trinidad of the rainfed South Asia rice breeding team of IRRI through the course of this study. The authors also thank E. Septiningsih, J. Vitto, and J. Carandang for help received in screening the lines for submergence tolerance.

Open Access This article is distributed under the terms of the Creative Commons Attribution 4.0 International License (http:// creativecommons.org/licenses/by/4.0/), which permits unrestricted use, distribution, and reproduction in any medium, provided you give appropriate credit to the original author(s) and the source, provide a link to the Creative Commons license, and indicate if changes were made.

\section{References}

Bernier J, Kumar A, Venuprasad R, Spaner D, Atlin GN (2007) A large-effect QTL for grain yield under reproductive-stage drought stress in upland rice. Crop Sci 47:507-516

Dilley M, Chen RS, Deichmann U, Lerner-Lam AL, Arnold M, Agwe J, Buys P, Kjekstad O, Lyon B, Yetman G (2005) 
Natural disaster hotspots: a global risk analysis synthesis report. Center for Hazard and Risk Research, Columbia University, New York

Dixit S, Biswal AK, Min A, Henry A, Oane RH, Raorane ML, Longkumer T, Pabuayon IM, Mutte SK, Vardarajan AR, Miro B, Govindan G, Albano-Enriquez B, Pueffeld M, Sreenivasulu N, Slamet-Loedin I, Sundarvelpandian K, Tsai Y-C, Raghuvanshi S, Hsing YC, Kumar A, Kohli A (2015a) Action of multiple intra-QTL genes concerted around a colocalized transcription factor underpins a large effect QTL. Nature Sci Rep 5:15183

Dixit S, Grondin A, Lee C-R, Henry A, Olds T-M, Kumar A (2015b) Understanding rice adaptation to varying agro-ecosystems: trait interactions and quantitative trait loci. BMC Genet 16:86. https://doi.org/10.1186/s12863-015-0249-1

Dixit S, Huang BE, Sta Cruz MT, Maturan PT, Ontoy JCE, Kumar A (2014a) QTLs for tolerance of drought and breeding for tolerance of Abiotic and biotic stress: an integrated approach. PLoS One 9(10):e109574

Dixit S, Singh A, Kumar A (2014b) Rice breeding for high grain yield under drought: a strategic solution to a complex problem. Int J Agron: Article ID 863683. https://doi.org/10.1155 $/ 2014 / 863683$

Dixit S, Singh A, Sta Cruz MT, Maturan PT, Amante M, Kumar A (2014c) Multiple major QTL lead to stable yield performance of rice cultivars across varying drought intensities. BMC Genet 15:16

Dixit S, Swamy BPM, Vikram P, Ahmed HU, Sta Cruz MT, Amante M, Atri D, Leung H, Kumar A (2012a) Fine mapping of QTLs for rice grain yield under drought reveals subQTLs conferring a response to variable drought severities. Theor Appl Genet 125:155-169

Dixit S, Swamy BPM, Vikram P, Bernier J, Sta Cruz MT, Amante M, Atri D, Kumar A (2012b) Increased drought tolerance and wider adaptability of $q D T Y 12.1$ conferred by its interaction with $q D T Y_{2.3}$ and $q D T Y_{3.2}$. Mol Breed 30:1767-1779

Ghimire KH, Quiatchon LA, Vikram P, Swamy BPM, Dixit S, Ahmed H, Hernandez JE, Borromeo TH, Kumar A (2012). Identification and mapping of a QTL $\left(q D T Y_{1.1}\right)$ with a consistent effect on grain yield under drought. Field Crops Res 131:88-96

Han F, Romagosa I, Ullrich SE, Jones BL, Hayes PM, Wesenberg DM (1997) Molecular marker-assisted selection for malting quality traits in barley. Mol Breed 3:427-437

Hattori Y, Nagai K, Furukawa S, Song X, Kawano R, Sakakibara $\mathrm{H}$, Wu J, Matsumoto T, Yoshimura A, Kitano H, Matsuoka M, Mori H, Ashikari M (2009) The ethylene response factors SNORKEL1 and SNORKEL2 allow rice to adapt to deep water. Nature 460:1026-1031

Henry A, Dixit S, Mandal NP, Anantha MS, Torres R, Kumar A (2014) Grain yield and physiological traits of rice lines with the drought yield QTL $q D T Y_{12.1}$ showed different responses to drought and soil characteristics in upland environments. Funct Plant Biol 41(11):1066-1077

Hittalmani S, Parco A, Mew TV, Zeigler RS, Huang N (2000) Fine mapping and DNA marker-assisted pyramiding of the three major genes for blast resistance in rice. Theor App Genet 100:1121-1128

Huang N, Angeles ER, Domingo J, Magpantay G, Singh S, Zhang G, Kumaravadivel N, Bennett J, Khush GS (1997) Pyramiding of bacterial blight resistance genes in rice: marker-assisted selection using RFLP and PCR. Theor App Gen 95:313-320

IRRI (International Rice Research Institute) (2014) Standard evaluation system for rice (SES), 5th edn. International Rice Research Institute, Los Baños

Joseph M, Gopalakrishnan S, Sharma RK, Singh VP, Singh AK, Singh NK, Mohapatra T (2004) Combining bacterial blight resistance and basmati quality characteristics by phenotypic and molecular marker-assisted selection in rice. Mol Breed 13:377-387

Komany S (2004) Progress of water environment governance in the Lao PDR -national strategy and adaptation program of action to climate change. Water Resources and Environment Agency, Lao

Kumar A, Dixit S, Henry A (2013) Marker-assisted introgression of major QTLs for grain yield under drought in rice. In: Varshney RK, Tuberosa R (eds) Translational genomics for crop breeding: abiotic stress, yield, and quality, 1st ed. John Wiley \& Sons Ltd, Chichester, pp 47-64

Kumar A, Dixit S, Ram T, Yadaw RB, Mishra KK, Mandal NP (2014) Breeding high-yielding drought-tolerant rice: genetic variations and conventional and molecular approaches. J Exp Bot 65(21):6265-6278

Kumar A, Verulkar SB, Dixit S, Chauhan B, Bernier J, Venuprasad R, Zhao D, Shrivastava MN (2009) Yield and yieldattributing traits of rice (Oryza sativa L.) under lowland drought and suitability of early vigor as a selection criterion. Field Crops Res 114:99-107

Linh LH, Linh TH, Xuan TD, Ham LH, Ismail AM, Khanh TD (2012) Molecular breeding to improve salt tolerance of rice (Oryza sativa L.) in the Red River Delta of Vietnam. Int J Plant Genet 2012: Article ID 949038. https://doi.org/10.1155 /2012/949038

Mishra KK, Vikram P, Yadaw RB, Swamy BPM, Dixit S, Sta Cruz MT, Maturan P, Marker S, Kumar A (2013) qDTY 12.1 : a locus with a consistent effect on grain yield under drought in rice. BMC Genet 14:12

Neeraja CN, Maghirang-Rodriguez R, Pamplona A, Heuer S, Collard BCY, Septiningsih EM, Vergara G, Sanchez D, Xu K, Ismail AM, Mackill DJ (2007) A marker-assisted backcross approach for developing submergence-tolerant rice cultivars. Theor Appl Genet 115:767-776

Palanog AD, Swamy BPM, Shamsudin NAA, Dixit S, Hernandez JE, Boromeo TH, Sta Cruz PC, Kumar A (2014) Grain yield QTLs with consistent-effect under reproductive-stage drought stress in rice. Field Crops Res 161:46-54

Sandhu N, Singh A, Dixit S, Sta Cruz MT, Maturan PC, Jain RK, Kumar A (2010) Identification and mapping of stable QTL with main and epistasis effect on rice grain yield under upland drought stress. BMC Genet 15:63. https://doi. org/10.1186/1471-2156-15-63

Septiningsih EM, Hidayatun N, Sanchez DL, Nugraha Y, Carandang J, Pamplona AM, Collard BCY, Ismail AM, Mackill DJ (2014) Accelerating the development of new submergence tolerant rice varieties: the case of CiherangSub1 and PSB Rc18-Sub1. Euphytica 202:259-268

Shamsudin NAA, Swamy BPM, Ratnam W, Sta. Cruz MT, Raman A, Kumar A (2016a) Marker assisted pyramiding of drought yield QTLs into a popular Malaysian rice cultivar, MR219. BMC Genet 17:30. https://doi.org/10.1186/s12863016-0334-0 
Shamsudin NAA, Swamy BPM, Ratnam W, Sta. Cruz MT, Sandhu N, Raman AK, Kumar A (2016b) Pyramiding of drought yield QTLs into a high-quality Malaysian rice cultivar MRQ74 improves yield under reproductive stage drought. Rice 9:21. https://doi.org/10.1186/s12284-0160093-6

Singh S, Sidhu JS, Huang N, Vikal Y, Li Z, Brar DS, Dhaliwal HS, Khush GS (2001) Pyramiding three bacterial blight resistance genes ( $x a 5$, xa13 and Xa21) using markerassisted selection into indica rice cultivar PR106. Theor App Genet 102:1011-1015

Swamy BPM, Ahmed HU, Henry A, Mauleon R, Dixit S, Vikram P, Tilatto R, Verulkar SB, Perraju P, Mandal NP, Variar M, Robin S, Chandrababu R, Singh ON, Dwivedi JL, Das SP, Mishra KK, Yadaw RB, Aditya TL, Karmakar B, Satoh K, Moumeni A, Kikuchi S, Leung H, Kumar A (2013) Genetic, physiological, and gene expression analyses reveal that multiple QTL enhance yield of rice mega-variety IR64 under drought. PLoS One 8(5):e62795

Toledo AMU, Ignacio JCI, Casal C Jr, Gonzaga ZJ, Mendioro MS, Septiningsih EM (2015) Development of improved Ciherang-Sub1 having tolerance to anaerobic germination conditions. Plant Breed Biotechnol 3(2):77-87

Uga Y, Sugimoto K, Ogawa S, Rane J, Ishitani M, Hara N, Kitomi Y, Inukai Y, Ono K, Kanno N, Inoue H, Takehisa H, Motoyama H, Nagamura Y, Wu J, Matsumoto T, Takai T, Okuno K (2013) Control of root system architecture by DEEPER ROOTING 1 increases rice yield under drought conditions. Nat Genet 45:1097-1102
Van Berloo R (2008) GGT 2.0: versatile software for visualization and analysis of genetic data. J Hered 99(2):232-236

Venuprasad R, Dalid CO, Del Valle M, Zhao D, Espiritu M, Sta Cruz MT, Amante M, Kumar A, Atlin GN (2009) Identification and characterization of large-effect quantitative trait loci for grain yield under lowland drought stress in rice using bulk-segregant analysis. Theor Appl Genet 120:177190

Vikram P, Swamy BPM, Dixit S, Ahmed HU, Sta Cruz MT, Singh AK, Kumar A (2011) $q D T Y_{1.1}$, a major QTL for rice grain yield under reproductive-stage drought stress with a consistent effect in multiple elite genetic backgrounds. BMC Genet $12: 89$

Xu K, Mackill DJ (1996) A major locus for submergence tolerance mapped on rice chromosome 9. Mol Breed 2:219-224

Yadav RB, Dixit S, Raman A, Mishra KK, Vikram P, Swamy BPM, Sta Cruz MT, Maturan PT, Pandey M, Kumar A (2013) A QTL for high grain yield under lowland drought in the background of popular rice variety Sabitri from Nepal. Field Crops Res 144:281-287

Yi M, New KT, Vanavichit A, Chai-arree W, Toojinda T (2009) Marker assisted backcross breeding to improve cooking quality traits in Myanmar rice cultivar Manawthukha. Field Crops Res 113:178-186

Zhang Y, Luo L, Xu C, Zhang Q, Xing Y (2006) Quantitative trait loci for panicle size, heading date and plant height cosegregating in trait-performance derived near-isogenic lines of rice (Oryza sativa). Theor Appl Genet 113:361-368 